\title{
Signal Acquisition and Dynamic Self-tuning Algorithm Implementation for Intelligent Instrument
}

\author{
Ying Lou ${ }^{1, a}$, Nan Wang ${ }^{2, b}$, Xuejie Wang ${ }^{3, c^{*}}$ \\ ${ }^{1}$ School of Electronic and Information Engineering,Liaoning Science and Technology \\ University,Anshan, 114000,China \\ ${ }^{2}$ Anshan power supply company ,Limited company of Liaoning province power, \\ Anshan,114001,China \\ ${ }^{3}$ School of Information \& Electrical Engineering,Zhejiang University City College, Hangzhou, \\ 310015,China \\ aemail:louying70@126.com, ${ }^{\text {bemail: xXB4922933@163.com, }{ }^{\mathrm{c} e m a i l}: x j w a n g @ z u c c . e d u . c n}$
}

\begin{abstract}
Keywords:Intelligent Instrument; Dynamic Self-tuning Algorithm;Reference Voltage
\end{abstract}
\begin{abstract}
The key problems of intelligent instrument in the signal acquisition process is a long-term stable and reliable operation level. The self-tuning algorithm proposed in this paper is a kind of intelligent instrument signal acquisition, solving linear error caused by system drift. The intelligent instrument can collect multichannel industrial common analog signal acquisition. Two different reference voltage should be collected for each channel signal, which is used to realize the selftuning function of the measurement in the instrument.
\end{abstract}

\section{Introduction}

Intelligent instrument is the instrument that applies artificial intelligent theory, method and technology, which make it with similar intelligent feature or function of human[1]. In order to implement this feature or function, intelligent instrument is no longer a simple hardware entity, but a combination of hardware and software with strong intelligence software and instrument software plays an important role in it[2,3]. Intelligent instrument has the following characteristics: gathering information, dialogue with the outside world, memory information, processing information, control, self calibration and self study[4]. Calibration self-learning of the intelligent instrument can adapt to the change of the outside world, for instance, it can automatically compensate the influence of environment change such as temperature, pressure and so on ,it often through the calibration (zero, gain, etc.) to ensure its accuracy. Thus intelligent instrument is mainly for data acquisition, data should be stored in the large capacity memory chips and can be displayed on the LCD screen with a variety of forms[5]. The instrument can also communicate with the upper machine through the RS485 or wireless network, realize the centralized management of the energy. In the process of signal acquisition, dynamic self-tuning will be completed based on the measurements of reference voltage and thus is the innovative and key technology of this intelligent instrument[6].

\section{Hardware Design}

The intelligent instrument can accomplish multichannel analog signal acquisition, the allowed input signals are $0 \sim 5 \mathrm{v}, 1 \sim 5 \mathrm{v}$, mv voltage signal ,0 $\sim 10 \mathrm{~mA}, 4 \sim 20 \mathrm{~mA}$ current signal, $E, J, K, S$, $R, T$ thermocouple signal and Pt100, Cu50, Cu100 thermal resistance signal. These signals can be adjusted to $0 \sim 500 \mathrm{mV}$ voltage signal by the conditioner module, and they will be sent to the $A / D$ conversion chip. The processor reads the converted digital signal, obtains sampling values by corresponding calculation formula for the different input signal. The conditioner module is made up of optical coupler relav capacitive switch digital ppotentiometer and other advanced components. Without changing the hardware, the processor can receive common industrial signals, change the circuit by changing the switch's state and change the magnification of the circuit by changing the digital potentiometer value through using software to set. Figure1 is thermal resistor input circuit. In 
the circuit, controllable switch has been substituted by switch resistance or wire, unused switch has been slightly, the processing of other signal input is only doing different control to switch in this circuit.

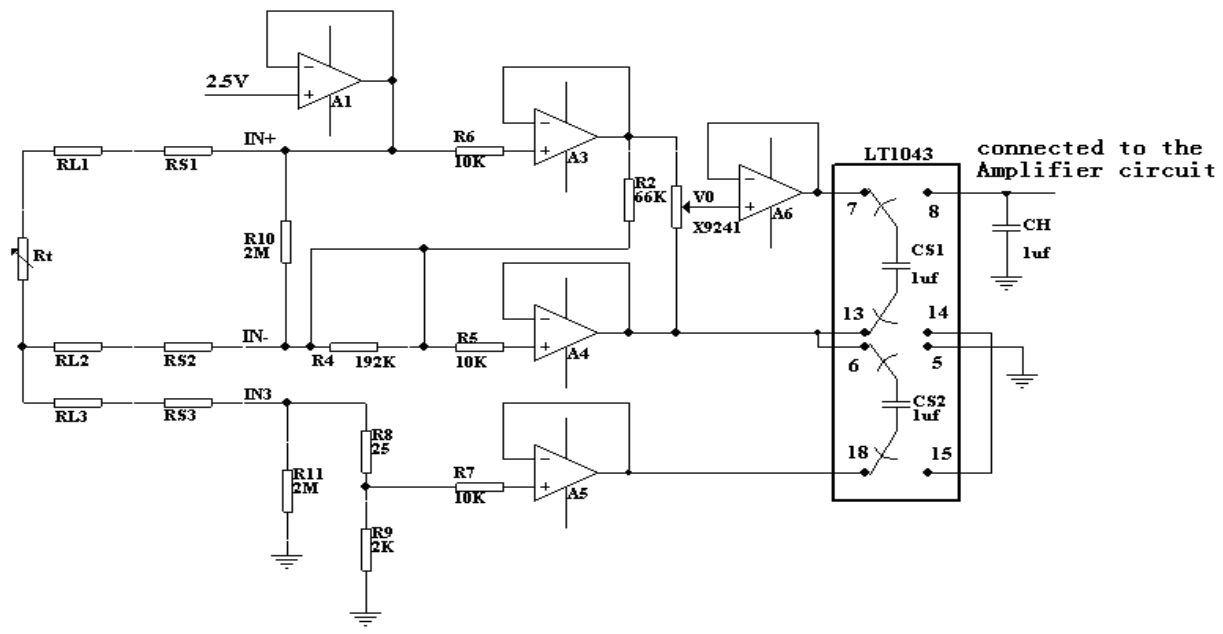

Figure.1.Thermal resistor input circuit

In the circuit, $R t$ is measuring thermal resistance, $R L 1, R L 2, R L 3$ are conductor resistances. This circuit can eliminate the effects of the wire resistance in long distance transmission by adapting three wire connection; $R S 1, R S 2, R S 3$ are the conduction resistances of the optical coupler relays.

Here analyze how to eliminate the error of the conductor resistance in the long distance transport by using the three wire connection. The reference voltage of value $2.5 \mathrm{~V}$ connects to the ground through the $R S 1, R L 1, R t, R L 3, R S 3, R 8$ and $R 9$, which converts input resistance to input voltage signal. Because the current in the R10 and R11 is very small, they can be ignored.

$V 12$ is the voltage between the $I N+$ and $I N-$, which connects to the two ends of the potentiometer VO through A3, A4 and get partial pressure VCS1 which connected to switch capacitance CS1,then

$$
V_{12}=2.5 * \frac{R L_{1}+R_{S 1}+R_{t}}{R_{S 1}+R_{L 1}+R_{t}+R_{L 3}+R_{8}+R_{9}}
$$

Which : $R a=R_{S 1}+R_{L 1}+R_{t}+R_{L 3}+R_{8}+R_{9}$

$$
V_{c s 1}=2.5 * \frac{k_{1} *\left(R_{L 1}+R_{S 1}+R_{t}\right)}{R_{a}}
$$

Note: the $K 1$ is the partial pressure ratio.

$V 23$ is the voltage between the output of the op-amp A4 and A5, which is connected to switch capacitance CS2, then the voltage of VCS2 is:

$$
V_{c s 2}=V_{23}=2.5 * \frac{R_{L 3}+R_{S 3}+R_{8}}{R_{a}}
$$

Because the pin 5 of $L T 1043$ is connected to the ground, pin14 and pin15 are short , so pin14 is - VCS2, then voltage between pin8 and pin14 is VCS1, then pin8' s potential to ground is VCS1 VCS2, it is also voltage on capacitors $\mathrm{CH}$ and named $\mathrm{Vch}$, so there is

$$
V_{c h}=V_{c s 1}-V_{c s 2}
$$

The formula (2 )and(3 )into the formula (4), we can obtain:

$$
V_{d}=\frac{25 * k_{1}^{*}\left(R_{\mathrm{L} 1}+R_{\mathrm{S1}}+R\right)-25^{*}\left(R_{\mathrm{LB}}+R_{\mathrm{S3}}+R_{8}\right)}{R_{a}}
$$


Because $R L 1=R L 3, R S 1=R S 3$, in order to eliminate their influence, $K 1$ should be 1 , so potentiometer $V 0$ ' s sliding side value should be setted to 63 , then

$$
V_{c h}=2.5 * \frac{R_{t}-R_{8}}{R_{a}}
$$

Seen from equation (6), by skillfully connection of LTC1043CN's two switch capacitor, the instrument can eliminate the influence of conductor resistance and resistance of optical coupler relay. The standard power supply is $2.5 \mathrm{~V}$, the precise resistance is $\mathrm{R} 8$, and they are affected by temperature changes slightly.In the Ra, precise resistance $R 9$ is $2 \mathrm{k}$, therefore the varies of conductor resistance and resistance of optical coupler relay with temperature changing will not have much impact on the measurement accuracy. Calculation is done according to the formula, when Pt100 is input, if the required temperature range is $0 \sim 850^{\circ} \mathrm{C}$, and $850^{\circ} \mathrm{C}$ is corresponded to $390.84 \mathrm{ohm}$, from the formula (6), Vck can be obtained about $376 \mathrm{mv}$, with the Vck the sliding values of digital potentiometer $V 1, V 2$, V3 can be calculated in the later amplifying circuit.For other types of heat resistance, the algorithm is basically the same. The corresponding relation curve between resistance and temperature is obtained through fitting, such as the calculation coefficient of Pt resistance is: $a=$ $3.90802 \mathrm{e}-03 ; b=5.802 \mathrm{e}-07$, the temperature calculation formula is:

$$
T=\frac{-a+\sqrt{a^{2}-4 * b * t_{1}}}{2 * b}
$$

Which $\mathrm{t} 1$ is the measured resistance value, $T$ is the corresponding temperature.

\section{Dynamic self correction algorithm}

In order to realize the correction function in the measurement of the instrument, the two different reference voltage need to be sampled before sampling each channel signal.Figure 2 is the reference voltage input circuit. MAX872 is a precise and standard power supply, which output is $2.5 \mathrm{~V}$. The voltage is divided by the precision resistor $R 1(66 \mathrm{k}), R 3(192 \mathrm{k})$, so we can get about $640 \mathrm{mv}$ and the voltage is connected to 0 potentiometer of digital potentiometer X9241.By adjusting the slide, it can output high and low two standard voltage source within the scope of 0 $500 \mathrm{mv}$. How to use the two standard source to realize dynamic calibration, the calibration algorithm is then given below.

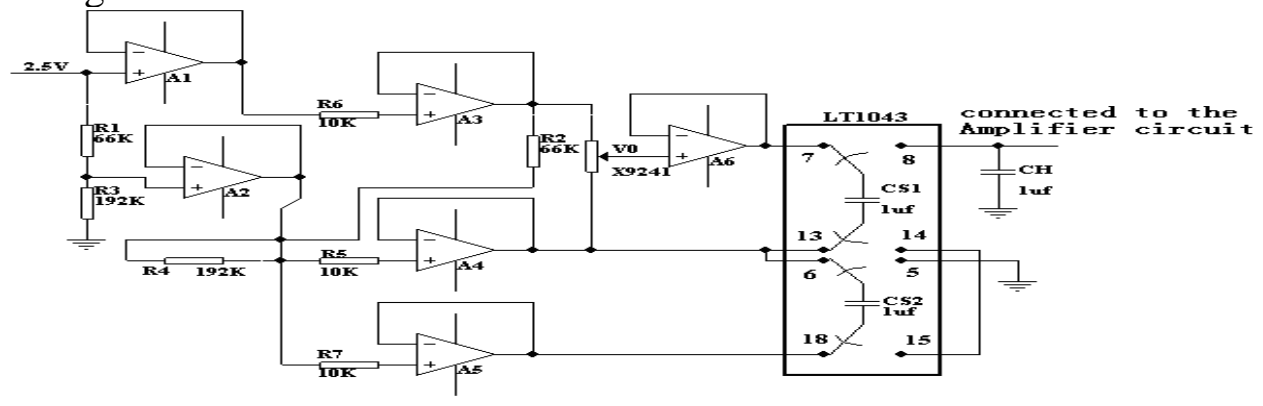

Figure 2. Reference voltage input circuit

Horizontal axis in the figure 2 represents the sample data, the vertical axis (or the corresponding theoretical sampling values)represents the engineering value. $Y h$ and $Y l$ are respectively as the upper limit and lower limit of the engineering value. When correcting the system, we can obtain $X h$ and $X l$ value by inputting $Y h$ and $Y l$, at the same time, $X t$ and $X s$ which are the sampled values of high and low two standard source are also obtained. Assumes that the instrument system is a linear system, so $A, B, D, E$ are in a straight line.According to the two point ,analytic equation can be written: 


$$
\frac{Y-Y l}{Y h-Y l}=\frac{X-X l}{X h-X l}
$$

So:

$$
\begin{aligned}
& Y=\frac{X-X l}{X h-X l}(Y h-Y l)+Y l \\
& =\frac{Y h-Y l}{X h-X l} X+\frac{Y l X h-Y h l}{X h-X l}
\end{aligned}
$$

Then according to the $X s$ and $X t$, their corresponding engineering values can be worked out:

$$
\begin{gathered}
Y t=\frac{Y h-Y l}{X h-X l} X t+\frac{Y l X h-Y h X l}{X h-X l} \\
Y s=\frac{Y h-Y l}{X h-X l} X s+\frac{Y l X h-Y h X l}{X h-X l}
\end{gathered}
$$

Then the correction of sampled data can be obtained by the line determined with the two point of $D$ and $E$. The equation can be written:

$$
\frac{Y-Y s}{Y t-Y s}=\frac{X-X s}{X t-X s} Y=\frac{Y t-Y s}{X t-X s} X+\frac{Y s X t-Y t X s}{X t-X s}
$$

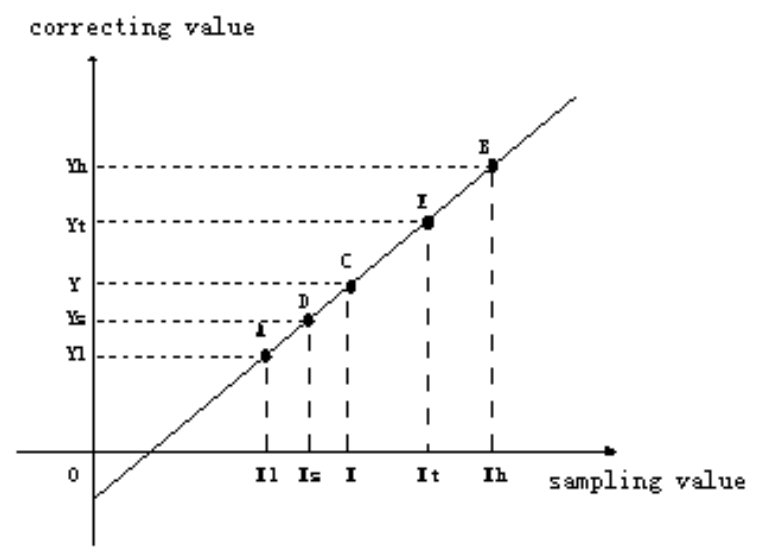

Figure.3. Correction algorithm schematic

The corrected engineering value $Y$ is calculated by using the sampled value $X$. In the above formula, $Y s$ and $Y t$ are constants, $X s=X s(t), X t=X t(t), X=X(t)$ is the function of time,so $Y=f(X)=Y(t)$ is also the function of time. If the system occurred linear drift, temperature drift or other linear changes in the system during the running process, the three time functions in the above will be changed in the same time. Due to that the changes in the results are all linear, thus $X$ will change to $k X+b$. So:

$$
\begin{aligned}
Y & =f(k X+b) \\
= & \frac{Y t-Y s}{k X t+b-(k X s+b)}(k X+b) \\
& +\frac{Y s(k X t+b)-Y t(k X s+b)}{k X t+b-(k X s+b)} \\
Y & =\frac{Y t-Y s}{X t-X s} X+\frac{Y s X t-Y t X s}{X t-X s}=f(X)
\end{aligned}
$$


It Can be seen from the above, when the system has linear deviation and slope deviation, it always has $Y=f(x)=f(k x+b)$,thus using the real-time dynamic correction algorithm can solve linear error caused by the system drift.

\section{Conclusion}

In the later measurements, even if linear changes have taken place in the system, the sampled values can be revised and get the correct actual theoretical value by the straight line equation. Through the aging test of high and low temperature and actual operation in the field , the results of these tests show that the calibration of intelligent methods has reached the design requirements, it can do much help to the long-term reliable operation of the intelligent instruments.

\section{Acknowledgement}

This paper is supported by Zhejiang province Higher Education Institute "125"educational science planning projects funding (KT2011356), Zhejiang University City College classroom teaching reform project "based on the idea of modeling and solution of model of project driving motor application technology " Fund (KG1203).

\section{References}

[1] X.F. Yao, and C.Y. Wang , "New Method of Online Configuration and Data Diagnosis Based on Intelligent Instrument,” Control and Instruments In Chemical Industry, vol.1, pp:65-68, 2011.

[2] X.C. Wu, and Y.Y. Yang, “ The design of parameters self-tuning PID fuzzy control in liquidlevel control system of double-tanks,” Automation \& Instrumentation, vol.4, pp:41-43, 2011.

[3] H.X. Yu, "Design of Intelligent Instrument Interface Circuit Functioning in Two Networks," Process Automation Instrumentation, vol.6, pp:73-75, .2011.

[4] J.L. Zhang, and B. Guang, "Research on Multichannel Test Signals and Data Acquisition and Processing System,” Computer Automated Measurement \& Control, vol. 10, pp:45-49, 2005.

[5] C.X. Ling, "Design and Realization of D istributed Control System Based on Intelligent Instrument,” Mechanical Engineering \& Automation, vol. 5, pp:124-126, 2010 .

[6] S. Anatoly, "Intelligent data acquisition and advanced computing systems," Computer Standards and Interfaces, vol. 24, pp:97-100, 2002. 